\title{
The association between oxidized low-density lipoprotein antibodies and hematological diseases
}

\author{
Hao Li ${ }^{1}$, Da-qing $L i^{2 *}$, Xiang-xin $\mathrm{Li}^{1}$ and Lu-qun Wang ${ }^{1}$
}

\begin{abstract}
Background: The aim of the study is to compare the profiles of antibodies (IgM and lgG) against oxidized low-density lipoprotein (oxLDL) of hematological diseases.

Methods: The serum antibodies of oxLDL-IgM and oxLDL-IgG for 446 cases with hematological diseases and 90 patients with primary hypertension and 90 healthy controls were measured by enzyme-linked immunosorbent assay (ELISA) in a cross-section survey. The association of serum oxLDL-LgM and oxLDL-IgG with hematological diseases was analyzed by multiple linear regression model.

Results: Comparing with the hypertension or normal groups, the levels of $T C H, T G, L D L-C, H D L-C$, oxLDL, and oxLDL-IgG were lower and the levels of ADP and oxLDL-IgM were higher in the hematological diseases group. The levels of oxLDL-IgG antibodies titer were different among hematological diseases group. The results of correlation and multiple regression analysis showed that the seven hematological disease subgroups were positively related to the oxLDL-IgM antibody titer but negatively related to the oxLDL-IgG antibody titer, having been adjusted for potential confounding factors such as age, SBP, DBP, BMI, TCH, TG, ADP, oxLDL, HDL-C, LDL-C.

Conclusions: Here we show that OxLDL-IgG antibodies titer were lower and of oxLDL-IgM titer were higher than hypertension and healthy individuals. Also oxLDL-lgG titer were different among hematological diseases group.
\end{abstract}

Keywords: Oxidized low-density lipoprotein, Autoantibody, Hematological diseases, oxLDL-lgG antibody, oxLDL-lgM antibody

\section{Background}

Oxidized low-density lipoprotein (oxLDL) consists of lipids, including cholesterol and oxysterols, phospholipids, oxidized fatty acids, aldehydes and lipid peroxides [1]. Studies have shown that oxLDL plays a major role in initiation and progression of atherosclerosis (AS) [2]. OxLDL has both proinflammatory and cytotoxic properties [3]. The relationship was firstly confirmed in the setting of hypercholesterolemia, with the highest IgM levels showing the lowest risk of coronary artery disease

\footnotetext{
*Correspondence: qingdali611@163.com

${ }^{2}$ The Key Laboratory of Cardiovascular Remodelling and Function Research, Chinese Ministry of Education and Chinese Ministry of Health, Department of Cardiology, Qilu Hospital, Shandong University, 107\# Wenhuaxi Road, Jinan 250012, China

Full list of author information is available at the end of the article
}

for the same level of hypercholesterolemia [4]. An epidemiological cohort of initially healthy subjects shows that IgG and IgM are not only independent predictors of coronary artery disease (CAD) events but also may modify CAD risk associated with elevated levels of oxidative biomarkers [5]. Interestingly, as early to be observed a high level of serum cholesterol may increase the risk of atherosclerosis, and a low level of serum cholesterol may increase the risk of cancer.

Recently, a genetic study showed the molecular basis of these connections, which indicated that lipid metabolism genes are important for transformation and are upregulated in cancer and atherosclerosis. The genes of activation oxLDL and its receptor are a molecular connection between cancer and atherosclerosis [6]. Natural 
antibodies play an important role in the process of homeostatic inflammation, cell death and apoptotic cell clearance [7]. Natural IgM antibodies (nIgM) can remove oxLDL. The levels of nIgM specific for phosphoryl choline (anti-PC), were significantly higher in rheumatoid arthritis (RA) patients than osteoarthritis (OA) patients $(P<0.05)$, and that implicates the deficiency in immune responses to oxLDL which may contribute to the metabolic syndromes in the development of OA [8]. Positive immunoglobulin (Ig)G anticardiolipin antibody (IgGaCL) was the main component of oxLDL antibody. One research showed that the IgGaCL was associated with splanchnic vein thrombosis [9]. Moreover, some investigators found that the roles of anti-OxLDL antibodies (IgG/ IgM) are related to atherosclerosis and relevant diseases [10-13]. The human natural monoclonal IgM antibody SAM-6 is related to apoptosis of cancer cells [14]. We found that a decreased oxLDL and oxLDL-lgG and the elevated oxLDL-lgM serum levels may be related to the development and progression of esophageal squamous cell carcinoma [15].

As far as we know, a few papers reported the relationship between lipid and leukemia diseases. One report showed that the serum cholesterol, LDL, and HDL are significantly reduced in myelodysplastic syndrome (MDS) patients [16]. Another report showed that chronic lymphocytic leukemia (CLL) cells are antigenexperienced which may be derived from self-reactive natural Ab-producing B cells [17]. Our previous work found a decreased oxLDL-IgG and an elevated oxLDL-IgM serum levels in adult with acute myeloblastic leukemia (AML) [18]. A study with very enlightening significance showed that a 54-year-old female patient who had systemic lupus erythematosus with marrow fibrosis, when treated with high dose of steroids and intravenous immunogloblulin got remission with normalization of the bone-marrow [19]. So far little information of oxLDL antibodies is available about hematological diseases, especially for leukemia.

The presence of oxLDL and autoantibodies in several hematological diseases, particularly in those with very poor prognosis, and the occurrence of these diseases is still not clear. The main goal of the present study is to investigate the profiles of oxLDL, oxLDL-IgG and oxLDL-Ig antibodies in some hematological diseases and compared with healthy and primary hypertension patients.

\section{Methods}

\section{Subjects}

We carried out a cross-sectional study including 286 cases that were first diagnosed according to the WHO diagnosis criteria during the period from Jan. 2008 to Feb. 2013 in the department of hematology, Qilu Hospital, Shandong University. Among them, 98 cases were diagnosed with non-lymphatic leukemia, 60 cases with lymphatic leukemia, 52 cases with hemolytic anemia, 66 cases with immunothrombocytopenia, 57 cases with multiple myeloma, 58 cases with non-Kochkin's lymphoma, 55 cases with myelodysplastic syndrome. Two groups were classified: the first group included 90 patients with primary hypertension diagnosed in the Division of Cardiology of the Qilu Hospital of Shandong University, and the second group comprised of 90 consecutive healthy normotensive persons serving as normal controls from a community screening examination of health care during the same period. Inclusion criteria were negative family history of CAD, myocardial infarction (MI), and stroke; nonsmoking status; and absence of hypercholesterolemia, hypertriglyceridemia, and diabetes mellitus. The Medical Ethics Committee of Qilu Hospital of Shandong University approved the study protocol, and written consent was obtained from each participant.

\section{Measurements}

A survey of the characteristics of the subjects, involving name, gender, age, occupation, etc., was conducted using a questionnaire. Blood pressure was measured by mercury sphygmomanometer using Korotkoff phase $\mathrm{V}$ for diastolic, according to the WHO guidelines. Hypertension was defined as systolic pressure $\geq 140 \mathrm{~mm} \mathrm{Hg}$ and/ or diastolic pressure $\geq 90 \mathrm{~mm} \mathrm{Hg}$ or use of any antihypertensive agents.

\section{Blood sampling and biochemical analysis}

Venous blood samples were taken in the morning after an overnight fast for at least $12 \mathrm{~h}$. The samples were taken from the femoral artery and immediately centrifuged at $3000 \mathrm{rpm}$ for $10 \mathrm{~min}$. The specimens were then frozen and kept at $-80{ }^{\circ} \mathrm{C}$ until assay. Total cholesterol (TCH), high density lipoprotein cholesterol (HDL-c), triglycerides (TG) levels were measured with conventional methods. Low density lipoprotein cholesterol (LDL-c) was calculated by the Fried Ewald formula (LDL-c) = $\mathrm{TCH}-(\mathrm{HDL}-\mathrm{c})-\mathrm{TG} / 5$. Serum adiponectin (ADP), oxLDL, oxLDL-IgG and oxLDL-IgM autoantibodies against-oxLDL measured by the enzyme-linked immunosorbent assay (ELISA) with commercially available kits (Adliteram diagnostic laboratories Ln., USA). The detail methods of oxLDL-IgG and oxLDLIgM measurements were shown in our previous reports $[15,18]$. OxLDL-lgG or oxLDL-lgM titers were calculated by constructing a standard curve according to the standards attached in the corresponding kits. These kits used the individually poached microplate strips that were coated with native or antigen; as a result, the antigen stability was shown to be longer than 4 months at least. In our work, the intra-assay 
and inter-assay reproducibility (coefficients of variation) of the assay were $3-5 \%$ and $4-7 \%$ respectively.

\section{Statistical analysis}

Shapiro-Wilk's W test of normality was used for data distribution analysis. The normality distributed data were expressed as the mean value $\pm \mathrm{SD}$. In the univariate analysis, multiple group comparisons were performed by one-way analysis of variance (ANOVA), and the significant differences between groups were assessed by Bonferroni test. A bivariate correlation analysis was conducted by the Spearman's rank method. A generalized linear regression model was analyzed considering the main effects of the oxLDL-IgM and oxLDL-IgG titer and covariates (confounding factors) including gender, age, body index (BMI), systolic blood pressure (SBP), diastolic blood pressure (DBP), TCH, TG, HDL-c, LDL-c, and ADP. The subgroup of the hematologic diseases was denoted as below: $1=$ cases with non-lymphatic leukemia, 2 = cases with lymphatic leukemia, 3 = cases with hemolytic anemia, $4=$ cases with immunothrombocytopenia, $5=$ cases with multiple myeloma, $6=$ cases with non Kochkin's lymphoma, $7=$ cases with myelodysplastic syndrome. The Levene test for homogeneity of variance and Bartlett's spherical test were carried out using the generalized linear model. In the analysis a two-tailed $P$ value $<0.05$ was considered as the statistical significance. All statistical analyses were done by SPSS19.0.

\section{Results}

We first measured the oxLDL-IgG and oxLDL-IgG antibody titers in a small pre-experiment. On the basis of these results, we calculated the sample size about $30 \sim$ 50 cases in each group so as to detect the main difference of each antibody titers with a statistical power of $9 \%$ at the 0.05 level (the calculation formula was $n=2\left(\left(\mathrm{t}_{\alpha / 2}+\right.\right.$ $\left.\left.\left.\mathrm{t}_{\beta}\right)^{\wedge}{ }^{2} \sigma^{\wedge}\right) / \delta^{\wedge}, \mathrm{t}_{\alpha / 2}=1.96, \beta=0.1, \sigma=9.0, \delta=6-8\right)$. The overall sample size of the study was at least 495 subjects. Finally we enrolled 626 subjects in this study.

Table 1 lists baseline characteristics of all subjects. The differences on the variables of age, systolic blood pressure and diastolic blood pressure are statistically significant among those groups. All these variables as covariates (confounding variables) are included in the multi linear regression model.

\section{Comparison of the levels of serum lipids between hypertension group and normal control group}

As shown by Tables 2 and 3, the levels of serum $\mathrm{TCH}$, TG, LDL-c, ADP and oxLDL-IgM in the hypertension group are higher than those in the normal control group. Comparing with the controls, the levels of HDLc, oxLDL and oxLDL-IgG in the hypertension group are lower, in particular the difference of oxLDL level is statistically significant $(P<0.005)$.

\section{Comparison of the levels of serum blood lipids between the hypertension group and the hematological diseases subgroups}

Comparing with the hypertension group (8), the levels of serum TCH, TG, LDL-c, HDL-c, oxLDL, oxLDL-IgG and oxLDL-IgM are lower in the seven subgroups of patients with hematological diseases. For oxLDL-IgM except the immunothrombocytopenia subgroup (4), the levels of oxLDL-IgM titer are significantly higher than that in the hypertension group, while the levels of serum oxLDL-IgG in the seven subgroups (from (1) to (7) shown in Table 2) of hematological diseases are lower than that in the hypertension group $(P<0.005)$.

Table 1 Demographic and clinical features of the subjects in the study

\begin{tabular}{llllll}
\hline Group & Gender $(M / F)$ & Age & BMl & SBP* & DBP+ \\
\hline cases with non lymphatic leukemia & $49 / 49$ & $43.2 \pm 18.18$ & $23.2 \pm 3.36$ & $126.0 \pm 16.33$ & $68.9 \pm 12.05$ \\
cases with lymphatic leukemia & $25 / 35$ & $31.1 \pm 15.40$ & $23.0 \pm 3.60$ & $123.1 \pm 9.30$ & $79.3 \pm 6.2$ \\
cases with Hemolytic anemia & $30 / 22$ & $50.5 \pm 20.40$ & $23.7 \pm 3.99$ & $133.8 \pm 19.62$ & $66.1 \pm 10.04$ \\
cases with immunothrombocytopenia & $25 / 41$ & $43.7 \pm 17.37$ & $22.5 \pm 3.11$ & $123.7 \pm 13.54$ & $76.9 \pm 9.99$ \\
cases with multiple myeloma & $32 / 25$ & $57.3 \pm 8.49$ & $24.9 \pm 2.73$ & $135.0 \pm 13.28$ & $81.3 \pm 12.24$ \\
cases with non Kochkin's & $22 / 36$ & $48.4 \pm 12.45$ & $26.6 \pm 4.36$ & $109.7 \pm 11.49$ & $73.6 \pm 8.97$ \\
cases with myelodysplastic syndrome & $25 / 30$ & $61.9 \pm 13.57$ & $25.1 \pm 3.67$ & $122.2 \pm 13.10$ & $67.7 \pm 10.15$ \\
patients with primary hypertension & $47 / 43$ & $56.5 \pm 10.87$ & $25.8 \pm 3.49$ & $159.2 \pm 17.15$ & $94.2 \pm 11.03$ \\
healthy normal persons+ & $40 / 50$ & $45.4 \pm 11.67$ & $24.5 \pm 3.35$ & $115.3 \pm 16.31$ & $78.2 \pm 10.03$ \\
$F / X^{2}$ & 10.724 & 16.662 & 4.766 & 40.045 & 30.959 \\
$P$ & 0.218 & $<0.001$ & 0.02 & $<0.001$ & $<0.001$ \\
\hline
\end{tabular}

*: SBP systoilic blood pressure, +: DBP diastolic blood pressure 
Table 2 The distribution of lipid profile in the nine sub groups of the study

\begin{tabular}{|c|c|c|c|c|c|c|c|c|}
\hline Serial number + group & $\mathrm{CTH}$ & TG & APD & $\mathrm{HDL}-\mathrm{C}$ & LDL-C & OxLDL & oxLDL-lgG & oxLDL-IgM \\
\hline (1) cases with non lymphatic leukemia & $4.00 \pm 1.018$ & $1.64 \pm 1.055$ & $7.56 \pm 9.976$ & $0.68 \pm 0.355$ & $2.97 \pm 0.991$ & $31.48 \pm 21.594$ & $38.57 \pm 19.483$ & $18.70 \pm 10.510$ \\
\hline (2) cases with lymphatic leukemia & $3.90 \pm 1.358$ & $1.44 \pm 0.910$ & $2.40 \pm 1.089$ & $1.14 \pm 0.834$ & $2.47 \pm 1.158$ & $21.76 \pm 10.846$ & $28.14 \pm 19.185$ & $20.24 \pm 9.915$ \\
\hline (3) cases with Hemolytic anemia & $3.07 \pm 0.805$ & $1.62 \pm 1.352$ & $6.58 \pm 8.409$ & $0.92 \pm 0.481$ & $2.13 \pm 1.079$ & $21.08 \pm 9.871$ & $31.98 \pm 20.364$ & $23.89 \pm 9.081$ \\
\hline (4) cases with immunothrombocytopenia & $4.50 \pm 0.866$ & $1.24 \pm 0.419$ & $4.59 \pm 6.395$ & $0.96 \pm 0.956$ & $3.28 \pm 1.047$ & $17.33 \pm 4.716$ & $45.96 \pm 25.804$ & $17.74 \pm 9.512$ \\
\hline (5) cases with multiple myeloma & $4.13 \pm 1.829$ & $1.60 \pm 0.903$ & $5.08 \pm 7.687$ & $0.44 \pm 0.312$ & $3.51 \pm 1.526$ & $25.39 \pm 12.593$ & $61.82 \pm 33.245$ & $17.97 \pm 6.918$ \\
\hline (6) cases with non Kochkin's & $5.50 \pm 1.273$ & $2.16 \pm 1.062$ & $5.49 \pm 8.018$ & $1.24 \pm 1.141$ & $3.82 \pm 1.073$ & $24.47 \pm 18.673$ & $38.25 \pm 19.241$ & $21.58 \pm 14.194$ \\
\hline (7) cases with myelodysplastic syndrome & $3.83 \pm 0.854$ & $1.19 \pm 0.352$ & $14.25 \pm 12.550$ & $0.70 \pm 0.799$ & $2.89 \pm 0.427$ & $52.68 \pm 33.884$ & $21.93 \pm 10.368$ & $23.17 \pm 12.745$ \\
\hline (8) patients with primary hypertension & $5.78 \pm 1.2223$ & $1.58 \pm 0.952$ & $5.57 \pm 7.457$ & $1.04 \pm 0.560$ & $4.43 \pm 1.401$ & $25.83 \pm 10.735$ & $82.18 \pm 10.117$ & $10.31 \pm 9.130$ \\
\hline (9) healthy normal persons & $5.09 \pm 0.568$ & $1.35 \pm 1.167$ & $3.28 \pm 4.068$ & $1.52 \pm 1.169$ & $3.36 \pm 1.281$ & $38.91 \pm 15.514$ & $77.59 \pm 7.165$ & $8.82 \pm 9.685$ \\
\hline $\mathrm{F}$ & 42.104 & 5.424 & 11.225 & 12.234 & 24.532 & 24.963 & 100.115 & 17.313 \\
\hline$P$ & $<0.001$ & $<0.001$ & $<0.001$ & $<0.001$ & $<0.001$ & $<0.001$ & $<0.001$ & $<0.001$ \\
\hline
\end{tabular}

Abbreviation: CTH total cholesterol, HDL-c high density lipoprotein cholesterol, TG triglycerides, LDL-c low density lipoprotein cholesterol, ADP Adiponectin 
Table 3 The post hoc Bonferroni's test for individual group-wise comparisons in Table 2

\begin{tabular}{|c|c|c|c|c|c|c|c|c|c|}
\hline (I) group & (J) group & $\mathrm{CTH}$ & TG & APD & $\mathrm{HDL}$ & LDL & oxLDL & oxLDL-lgG & oxLDL-IgM \\
\hline \multirow[t]{8}{*}{ (1) } & (2) & 1.000 & 1.000 & 0.002 & 0.015 & 0.305 & 0.020 & 0.032 & 1.000 \\
\hline & (3) & $<0.001$ & 1.000 & 1.000 & 1.000 & 0.001 & 0.015 & 1.000 & 0.144 \\
\hline & (4) & 0.247 & 0.339 & 0.638 & 1.000 & 1.000 & $<0.001$ & 0.545 & 1.000 \\
\hline & (5) & 1.000 & 1.000 & 1.000 & 1.000 & 0.217 & 1.000 & $<0.001$ & 1.000 \\
\hline & (6) & $<0.001$ & 0.046 & 1.000 & 0.001 & $<0.001$ & 0.491 & 1.000 & 1.000 \\
\hline & (7) & 1.000 & 0.192 & $<0.001$ & 1.000 & 1.000 & $<0.001$ & $<0.001$ & 0.417 \\
\hline & (8) & $<0.001$ & 1.000 & 1.000 & 0.075 & $<0.001$ & 0.858 & $<0.001$ & $<0.001$ \\
\hline & (9) & $<0.001$ & 1.000 & 0.020 & $<0.001$ & 0.903 & 0.111 & $<0.001$ & $<0.001$ \\
\hline \multirow[t]{7}{*}{ (2) } & (3) & 0.003 & 1.000 & 0.183 & 1.000 & 1.000 & 1.000 & 1.000 & 1.000 \\
\hline & (4) & 0.137 & 1.000 & 1.000 & 1.000 & 0.004 & 1.000 & $<0.001$ & 1.000 \\
\hline & (5) & 1.000 & 1.000 & 1.000 & $<0.001$ & $<0.001$ & 1.000 & $<0.001$ & 1.000 \\
\hline & (6) & $<0.001$ & 0.002 & 1.000 & 1.000 & $<0.001$ & 1.000 & 0.058 & 1.000 \\
\hline & (7) & 1.000 & 1.000 & $<0.001$ & 0.101 & 1.000 & $<0.001$ & 1.000 & 1.000 \\
\hline & (8) & $<0.001$ & 1.000 & 0.569 & 1.000 & $<0.001$ & 1.000 & $<0.001$ & $<0.001$ \\
\hline & (9) & $<0.001$ & 1.000 & 1.000 & 0.186 & $<0.001$ & $<0.001$ & $<0.001$ & $<0.001$ \\
\hline \multirow[t]{6}{*}{ (3) } & (4) & $<0.001$ & 1.000 & 1.000 & 1.000 & $<0.001$ & 1.000 & 0.003 & 0.057 \\
\hline & (5) & $<0.001$ & 1.000 & 1.000 & 0.056 & $<0.001$ & 1.000 & $<0.001$ & 0.118 \\
\hline & (6) & $<0.001$ & 0.146 & 1.000 & 1.000 & $<0.001$ & 1.000 & 1.000 & 1.000 \\
\hline & (7) & 0.017 & 0.672 & $<0.001$ & 1.000 & 0.027 & $<0.001$ & 0.237 & 1.000 \\
\hline & (8) & $<0.001$ & 1.000 & 1.000 & 1.000 & $<0.001$ & 1.000 & $<0.001$ & $<0.001$ \\
\hline & (9) & $<0.001$ & 1.000 & 1.000 & 0.001 & $<0.001$ & $<0.001$ & $<0.001$ & $<0.001$ \\
\hline \multirow[t]{5}{*}{ (4) } & (5) & 1.000 & 1.000 & 1.000 & 0.011 & 1.000 & 0.339 & $<0.001$ & 1.000 \\
\hline & (6) & $<0.001$ & $<0.001$ & 1.000 & 1.000 & 0.330 & 0.746 & 1.000 & 1.000 \\
\hline & (7) & 0.048 & 1.000 & $<0.001$ & 1.000 & 1.000 & $<0.001$ & $<0.001$ & 0.167 \\
\hline & (8) & $<0.001$ & 1.000 & 1.000 & 1.000 & $<0.001$ & 0.082 & $<0.001$ & 0.005 \\
\hline & (9) & 0.034 & 1.000 & 1.000 & 0.001 & 1.000 & $<0.001$ & $<0.001$ & $<0.001$ \\
\hline \multirow[t]{4}{*}{ (5) } & (6) & $<0.001$ & 0.074 & 1.000 & $<0.001$ & 1.000 & 1.000 & $<0.001$ & 1.000 \\
\hline & (7) & 1.000 & 0.842 & $<0.001$ & 1.000 & 0.179 & $<0.001$ & $<0.001$ & 0.315 \\
\hline & (8) & $<0.001$ & 1.000 & 1.000 & $<0.001$ & $<0.001$ & 1.000 & $<0.001$ & 0.006 \\
\hline & (9) & $<0.001$ & 1.000 & 1.000 & $<0.001$ & 1.000 & $<0.001$ & $<0.001$ & $<0.001$ \\
\hline \multirow[t]{3}{*}{ (6) } & (7) & $<0.001$ & $<0.001$ & $<0.001$ & 0.011 & 0.001 & $<0.001$ & $<0.001$ & 1.000 \\
\hline & (8) & 1.000 & 0.014 & 1.000 & 1.000 & 0.074 & 1.000 & $<0.001$ & $<0.001$ \\
\hline & (9) & 1.000 & $<0.001$ & 1.000 & 1.000 & 0.625 & $<0.001$ & $<0.001$ & $<0.001$ \\
\hline \multirow[t]{2}{*}{ (7) } & (8) & $<0.001$ & 0.639 & $<0.001$ & 0.443 & $<0.001$ & $<0.001$ & $<0.001$ & $<0.001$ \\
\hline & (9) & $<0.001$ & 1.000 & $<0.001$ & $<0.001$ & 0.694 & $<0.001$ & $<0.001$ & $<0.001$ \\
\hline (8) & (9) & 0.001 & 1.000 & 1.000 & 0.002 & $<0.001$ & $<0.001$ & 1.000 & 1.000 \\
\hline
\end{tabular}

Note: (1),(2),(3),(4),(5,(6,(7),(8),(9) is the serial number of each subgroup indicator in Table 2

Comparison of the levels of serum blood lipids between the normal control group and the hematological diseases subgroups

In the Tables 2 and 3 are the results of multiple group comparison and post hoc Bonferroni's test for individual group-wise comparison of the profiles of lipid in the study. Compared with (9, the levels of oxLDL-IgG antibodies titer significantly increased, and the oxLDLIgM titer decreased, and except (6), plasma CTH levels significant decline in the subsets (from (1) to (7)) of the hematological diseases group $(P<0.001)$. Except (1), plasma oxLDL levels in (7) was higher and in the others subsets of the hematological diseases group was lower than in (9) $(P<0.05)$. 


\section{Comparison of the levels of serum blood lipids in the seven subgroups of hematological diseases}

In the seven hematological diseases group the levels of oxLDL-IgG antibodies titer were statistically significant in between-group comparisons including (1)-(2), (1)-(3), (2)-(5), (3)-(4), (3)-(5), (4)-(5), (4)-(7), (5)-(6) and (5)-(7) $(P<$ 0.05); However, there was no significant difference for the levels of oxLDL-IgM antibodies titer $(P>0.05)$. Plasma oxLDL levels were statistically significant in between subsets comparisons including (1)-(2), (1)-(3), (2)-(7), (3)-(7), (3)-(7), (4)-(7) and (5)-(7) $(P<0.05)$.

A bivariate correlation analysis (Table 4) shows that the oxLDL-IgG antibody titer is positively related to age, BMI, systolic blood pressure (SBP), diastolic blood pressure (DBP), TCH, LDL-c and oxLDL but inversely related to adiponectin (ADP) and triglyceride (TG); Moreover, the levels of oxLDL-IgM antibody titer are inversely related to age, BMI, DBP, TCH and LDL-C and positively related to ADP and TG. It is noteworthy that there is an inversely relationship between the levels of oxLDL-IgM titer and the levels of oxLDL-IgM titer $(r=-0.368, P<0.01)$.

Table 5 lists the correlation coefficients between the levels of serum blood lipid (including TCH, TG, LDL-C HDL-c, oxLDL and ADP) and the oxLDL-IgM /oxLDLIgG antibodies titer. In the hypertension group, the levels of serum oxLDL-IgM titer are positively related to ADP and TG and inversely related to the levels of serum oxLDL; the levels of oxLDL-IgG antibody titer are positively related to the levels of serum LDL-c and $\mathrm{TCH}$.

In the normal control group the levels of serum oxLDL-IgM antibody titer are positively related to LDLc, ADP and TG and inversely related to the levels of serum HDL-c; however, the levels of serum oxLDL-IgG antibody titer are positively related to the levels of serum HDL-c and inversely related to the levels of serum ADP.

In the seven subgroups of hematological diseases the tendency of correlation is quite different. For example, in the non-lymphatic leukemia group, the level of the oxLDL-IgM antibody titer is positively related to the levels of serum TCH, oxLDL-c and ADP but inversely related to the levels of serum LDL-c; however, there is not significant correlation between oxLDL-IgG antibody titer among those study groups.

\section{Multiple linear regression model analysis}

Table 6 lists that the outcome of two multiple linear regression model analyses, with the dependent variable of the oxLDL-IgM and oxLDL-IgG antibody titer respectively. Comparing with the normal controls, 7 subgroups of hematological diseases have the positive relationship to the levels of serum oxLDL-IgM antibody titers but the negative relationship to the levels of serum oxLDL-IgG antibody titers, having been adjusted for potential confounding variables such as age, SBP, DBP, BMI, TCH, TG, ADP, oxLDL, HDL-c, LDL$\mathrm{C}$ and hypertension group.

\section{Discussion}

In the present study, we found out that (1) the profiles of serum lipid including oxLDL-IgM and oxLDL-IgG are consistent with the reported results in the literature $[4,5]$, comparing with the normal controls; (2) the mean levels of the serum lipid including TCH, TG, LDL-c, HDL-c, oxLDL, oxLDL-IgG and oxLDL-IgM are lower and the levels of ADP and oxLDL-IgM are higher in the patients with seven types of hematological diseases, comparing with normal controls; (3) the change in the trend of the

Table 4 The correlation coefficients of the measurement variables in the study

\begin{tabular}{|c|c|c|c|c|c|c|c|c|c|c|c|c|c|}
\hline & Group & oxLDL-IgM & oxLDL-IgG & LDL-C & $\mathrm{HDL}-\mathrm{C}$ & OxLDL & ADP & $\mathrm{TG}$ & $\mathrm{TCH}$ & SBP & DBP & $\mathrm{BMI}$ & Age \\
\hline$\overline{\text { Group }^{a}}$ & 1.000 & & & & & & & & & & & & \\
\hline oxLDL-IgM & $-0.343^{* *}$ & 1.000 & & & & & & & & & & & \\
\hline oxLDL-IgG & $0.494^{* *}$ & $-0.368^{* *}$ & 1.000 & & & & & & & & & & \\
\hline$L D L-C$ & $0.293^{* *}$ & $-0.300^{* *}$ & $0.301^{* *}$ & 1.000 & & & & & & & & & \\
\hline $\mathrm{HDL}-\mathrm{C}$ & $0.169^{* *}$ & -0.084 & -0.024 & $-0.225^{* *}$ & 1.000 & & & & & & & & \\
\hline OxLDL & $0.187^{* *}$ & -0.008 & $0.237^{* *}$ & -0.058 & 0.090 & 1.000 & & & & & & & \\
\hline ADP & $-0.182^{* *}$ & $0.482^{* *}$ & $-0.199^{* *}$ & $-0.182^{* *}$ & 0.002 & 0.062 & & & & & & & \\
\hline TG & -0.048 & -0.031 & $-0.157^{* *}$ & $0.302^{* *}$ & $-0.221^{* *}$ & -0.085 & -0.050 & 1.000 & & & & & \\
\hline $\mathrm{TCH}$ & $0.417^{* *}$ & $-0.379^{* *}$ & $0.287^{* *}$ & $0.802^{* *}$ & $0.239^{* *}$ & 0.046 & $-0.213^{* *}$ & $0.299^{* *}$ & 1.000 & & & & \\
\hline SBP & 0.051 & -0.022 & $0.248^{* *}$ & $0.106^{*}$ & 0.030 & 0.014 & 0.014 & 0.022 & $0.102^{*}$ & 1.000 & & & \\
\hline DBP & $0.367^{* *}$ & $-0.184^{* *}$ & $0.390^{* *}$ & $0.312^{* *}$ & $0.106^{*}$ & 0.022 & $-0.184^{* *}$ & $0.204^{* *}$ & $0.432^{* *}$ & $0.557^{* *}$ & 1.000 & & \\
\hline BMl & $0.225^{* *}$ & $-0.145^{* *}$ & $0.227^{* *}$ & $0.246^{* *}$ & -0.035 & 0.003 & -0.025 & $0.219^{* *}$ & $0.282^{* *}$ & $0.205^{* *}$ & $0.302^{* *}$ & 1.000 & \\
\hline Age & $0.258^{* *}$ & $-0.137^{* *}$ & $0.142^{* *}$ & $0.156^{* *}$ & $-0.111^{*}$ & -0.099 & -0.091 & -0.003 & $0.122^{*}$ & $0.315^{* *}$ & $0.108^{*}$ & $0.120^{*}$ & 1.000 \\
\hline Gender & -0.005 & -0.056 & 0.003 & $0.124^{*}$ & $0.211^{* *}$ & -0.087 & -0.054 & -0.078 & $0.211^{* *}$ & $-0.135^{* *}$ & 0.086 & -0.021 & -0.099 \\
\hline
\end{tabular}

${ }^{a}$ The variable group consists of nine sub groups that listed in the Table 4; $*: P<0.05, * *: P<0.01$ 
Table 5 The correlation coefficients between blood lipid terms and XLDL-lgG/oxLDL-IgM antibodies titer in each sub-groups

\begin{tabular}{|c|c|c|c|c|c|c|c|c|}
\hline Group & & $\lg G$ & LDL-C & $\mathrm{HDL}-\mathrm{C}$ & OXLDL & ADP & $\mathrm{TCH}$ & TG \\
\hline \multirow[t]{2}{*}{ cases with non lymphatic leukemia, } & $\lg M$ & -0.063 & $-0.259^{* *}$ & -0.099 & $0.267^{* *}$ & $0.473^{* *}$ & $-0.239^{*}$ & -0.176 \\
\hline & $\lg G$ & 1.000 & 0.133 & -0.180 & 0.013 & -0.082 & 0.003 & -0.149 \\
\hline \multirow[t]{2}{*}{ cases with lymphatic leukemia } & $\lg M$ & -0.071 & 0.067 & $0.421^{*}$ & -0.168 & $0.392^{*}$ & 0.244 & -0.125 \\
\hline & $\lg G$ & 1.000 & 0.110 & -0.201 & $0.627^{* *}$ & -0.128 & -0.306 & 0.107 \\
\hline \multirow[t]{2}{*}{ cases with hemolytic anemia } & $\lg M$ & -0.246 & $-0.611^{* *}$ & $0.425^{*}$ & -0.156 & 0.073 & $-0.832^{* *}$ & 0.374 \\
\hline & $\lg G$ & 1.000 & 0.226 & 0.140 & $0.911^{* *}$ & -0.112 & $0.436^{*}$ & -0.358 \\
\hline \multirow[t]{2}{*}{ cases with immunothrombocytopenia } & $\lg M$ & 0.067 & -0.233 & -0.167 & $0.517^{* *}$ & $0.350^{*}$ & $-0.600^{* *}$ & -0.267 \\
\hline & $\lg G$ & 1.000 & 0.233 & $-0.800^{* *}$ & -0.300 & 0.100 & $-0.383^{*}$ & $-0.400^{*}$ \\
\hline \multirow[t]{2}{*}{ cases with multiple myeloma } & $\lg M$ & -0.263 & $0.408^{*}$ & $0.490^{*}$ & -0.207 & $0.503^{* *}$ & $0.408^{*}$ & $0.531^{*}$ \\
\hline & $\lg G$ & 1.000 & $-0.944^{* *}$ & $-0.524^{* *}$ & 0.296 & -0.056 & $-0.944^{* *}$ & $-0.821^{*}$ \\
\hline \multirow[t]{2}{*}{ cases with non Kochkin's lymphoma } & $\lg M$ & $-0.417^{*}$ & $-0.517^{* *}$ & $0.683^{* *}$ & $0.383^{*}$ & $0.351^{*}$ & -0.183 & $-0.433^{*}$ \\
\hline & $\lg G$ & 1.000 & $0.400^{*}$ & $-0.533^{* *}$ & $0.450^{* *}$ & -0.243 & -0.083 & -0.067 \\
\hline \multirow[t]{2}{*}{ cases with myelodysplastic syndrome } & $\lg M$ & 0.264 & -0.178 & -0.067 & $0.584^{* *}$ & $0.962^{* *}$ & -0.042 & $-0.660^{*}$ \\
\hline & $\lg G$ & 1.000 & $-0.624^{* *}$ & $0.529^{* *}$ & 0.257 & 0.222 & $0.363^{*}$ & -0.186 \\
\hline \multirow[t]{2}{*}{ patients with primary hypertension } & $\lg M$ & 0.213 & -0.198 & 0.168 & $-0.273^{*}$ & $0.433^{* *}$ & -0.141 & $0.296^{*}$ \\
\hline & $\lg G$ & 1.000 & $0.320^{*}$ & -0.153 & -0.065 & 0.030 & $0.276^{*}$ & 0.094 \\
\hline \multirow[t]{2}{*}{ healthy normal control } & $\lg M$ & -0.131 & $0.455^{* *}$ & $-0.396^{* *}$ & 0.193 & $0.449^{* *}$ & 0.031 & $0.390^{*}$ \\
\hline & $\lg G$ & 1.000 & -0.198 & $0.330^{*}$ & -0.010 & $-0.499^{* *}$ & 0.015 & -0.212 \\
\hline
\end{tabular}

${ }^{*}: P<0.05,{ }^{* *}: P<0.01$

profiles of serum lipid is the same as that of the normal controls, comparing with the patients with hypertension. The results suggest that the association tendency between lipids and cardiovascular diseases is different from that between lipid and hematological diseases. At the same time the levels of oxLDL-IgG have a significant difference among the different hematological diseases, in particular for leukemia. This result is consistent with our previous report [18].
The immunoglobulin, the oxLDL-IgM and oxLDL-IgG antibodies, are the origin of autoantibody production of oxLDL. Therefore, it is reasonable to conclude that they probably play a more important physiological role in modulating the development of hematological diseases. In view of the molecular immunology, it's well known that the molecule of the IgM antibody is relatively large and responds sensitively to the recent infectious agent; however, the molecule of the IgG antibody is relatively

Table 6 The main effects for the variables related to the dependent variable of oxLDL-lgM or oxLDL-lgG in the generated linear regression models ${ }^{b}$

\begin{tabular}{|c|c|c|c|c|c|c|}
\hline \multirow[t]{2}{*}{ Parameters in the regression model } & \multicolumn{3}{|c|}{ Dependent Var oxLDL-IgM } & \multicolumn{3}{|c|}{ Dependent Var oxLDL-IgG } \\
\hline & $B$ & Wald & $P$ & B & Wald & $P$ \\
\hline Constant & 13.281 & 7.294 & 0.007 & 69.359 & 52.451 & $<0.001$ \\
\hline Gender (M/F) & 0.054 & 0.003 & 0.956 & 5.797 & 8.663 & 0.003 \\
\hline cases with non lymphatic leukemia & 5.418 & 7.315 & 0.007 & -37.985 & 106.186 & $<0.001$ \\
\hline cases with lymphatic leukemia & 8.340 & 11.736 & 0.001 & -47.171 & 110.637 & $<0.001$ \\
\hline cases with Hemolytic anemia & 11.541 & 18.090 & $<0.001$ & -44.355 & 71.523 & $<0.001$ \\
\hline cases with immunothrombocytopenia & 6.469 & 9.687 & 0.002 & -26.003 & 39.866 & $<0.001$ \\
\hline cases with multiple myeloma & 8.833 & 15.391 & $<0.001$ & -16.732 & 13.011 & $<0.001$ \\
\hline cases with non Kochkin's & 9.716 & 22.301 & $<0.001$ & -32.233 & 63.987 & $<0.001$ \\
\hline cases with myelodysplastic syndrome & 7.196 & 7.659 & 0.006 & -57.709 & 161.408 & $<0.001$ \\
\hline patients with primary hypertension & 1.692 & 0.697 & 0.404 & 4.992 & 1.440 & 0.230 \\
\hline healthy normal persons ${ }^{a}$ & 0.0 & . & . & 0.0 & & . \\
\hline
\end{tabular}

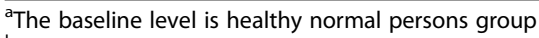

${ }^{b}$ In the two regression models we adjusted for potential confounding factors including age, SBP, DBP, BMI, TCH, TG, ADP, oxLDL, HDL-C, LDL-C 
small and responds sensitively to the previous infectious agent, indicating the host with an ability of immunity to the agent.

To the best of our knowledge, no related work has been reported previously. In our previous studies we found that the cytotoxicity of oxLDL on vascular endothelial cells line was lower than that on esophageal cancer cell and leukemia cell lines, as well as in patients with squamous cell esophageal carcinoma with a higher level of the oxLDL-IgM antibody titer and lower level of the oxLDL-IgG antibody titer, comparing with the normal persons $[15,18,20]$. The concentrations of ox-LDL required to induce apoptosis are usually $>20 \mu \mathrm{g} / \mathrm{m}$; these concentrations are quite high and observed only in patients with acute coronary syndromes [21]. Low physiologic concentrations $(<5 \mu \mathrm{g} / \mathrm{ml})$ do not cause significant apoptosis, and are associated with the cell proliferation [22]. Our assay indicated that the cytotoxic effects of oxLDL on K562/AO2 and EC-9706 were significantly higher than that on cancer cells of HT29, OVCAR3 and OVCAR5, HeLa, MCF7, A549 and PC3, when all aforementioned cell lines treated by oxLDL with the doses ranging from 100 to $300 \mu \mathrm{g} / \mathrm{ml}$ inhibited cell proliferations at dose- and time- dependence [23]. Combined with the results of the present study, we put forward the following hypothesis: oxLDL inducing autoimmune response is not only involved in the development of atherosclerosis or other vascular disease, but also related in the process of cancer and other chronic diseases including hematological diseases.

Oxidation of lipoproteins and oxidative processes generally play an important role in the initiation and progression of atherosclerotic lesions and the main mechanism is associated with the inflammatory and immune responses [24]. Recently, Lopes-Virella MF \& Virella G presented a review of the modified LDL including oxLDL auto antibodies in relation of the adaptive immune response in atherosclerosis (AS) [25]. We reviewed the literature reports and divided them into three categories: first hypothesis, oxLDL antibodies as an independent factors doctrine, some reports indicated that oxLDL and its autoantibody oxLDL-IgG can increase the risk of AS, but its autoantibody oxLDL-IgG may decrease the risk of AS [26]; Second hypothesis, some reports contradicted this conclusion [27], Third hypothesis, some reports suggested a humoral immune response to the modified LDL (mLDL) can be pathogenic rather than the protective factor $[25,28]$. One relatively large sample clinic data showed that the titer levels of antibodies to oxLDL in the CAD patients without and with acute coronary syndromes had not significant differences [29]. So far, the mechanism of antibodies of oxLDL or other mLDL associated with AS is still unclear. The evidences supporting oxLDL antibodies as the independent predictor for AS are summarized as following:First, the IgG and IgM antibodies provide directly protective effect due to the combining immune complexes [30]. Second, the antibodies to oxLDL block the uptake of oxLDL by macrophages and prevent the foam cell formation [28]. Third, probably the oxLDLAbs directed against some oxidized-phospholipid epitopes but not against others play a major protective role [31]. Once activated oxLDL antigen, $\mathrm{T}$ cells secrete a variety of cytokines and Th1 and Th2 cells have been postulated to play different roles in promoting inflammatory response [32].

The IgG and IgM antibodies are produced mainly due to the modification forms of oxLDL, malondialdehydemodified LDL (MDA-LDL) and advanced glycosylation end product-modified LDL (AGE-LDL) [32]. Freigang S. et al. found that the antiatherogenic effect of immunization of modified LDL is not primarily dependent on very high titers of antibodies but more likely to result from the activation of cellular immune responses [26]. Recently, the results of some studies also showed that oxLDL auto antibody can be the cellular immune responses in the pathogenic process of diseases [33-35]. OxLDL was lower, whereas oxLDL-Ab was higher in patients without cardiovascular disease (CVD) comparing with those with CVD [33]. The blood level of assessed antibodies was significantly higher in stroke patients than in control group. However, this phenomenon does not seem to protect patients against cerebrovascular events [34]. Recently a report found that a quiescent population of IgM+ plasma cells including oxLDL-specific IgM antibody secreting cells in bone marrow also sustained the elevated IgM antibody response in circulation [35]. One author reported that comparing with the patients (aged $>65$ years) with OxLDL-Ab level $\geq 200$ arbitrary U/ml, the Ox LDL-Ab level $<200$ arbitrary U/ml Adjusted Hazard Ratio (HR) was 2.67 (95 \% CI 1.58-4.52) for composite outcome (hospitalization and mortality). OxLDL-Ab level was the best indicator for both hospitalization and composite outcome [36]. It is excited that a new approach of determination OXLDL antibody component, a novel single-chain variable fragments (scFvs) $\gamma \kappa 5$, has been established and the $\gamma \kappa 5 \mathrm{scFv}$ can detect the modified LDL at very low concentrations [37]. This will promote the deep study of the relationship between oxLDL antibodies and diseases.

The main limitation of this study is due to small sample of patients with hematological diseases in cross-section design, and still needs a large sample in prospective study to get a strong evidence to support the idea. Also the mechanism of the oxLDL and its auto-antibodies involved the development of various hematological diseases needs to be elucidated in the future.

\section{Conclusions}

Our data suggest that the trend of association between lipids and cardiovascular diseases is different from that 
between lipid and hematological diseases. Also that the common characters of the different hematological diseases with the oxLDL-IgG antibody titers were lower, while the oxLDL-IgM antibody titers were higher than the normal group. Finally there were statistically significant differences of the oxLDL-IgG antibody titers among those hematological diseases. The results of this study are useful for further study on etiology and serious complications of hematological diseases.

\author{
Abbreviations \\ ADP: Adiponectin; AS: Atherosclerosis; CAD: Coronary artery disease; \\ CTH: Total cholesterol; CVD: Cardiovascular disease; HDL-c: High density \\ lipoprotein cholesterol; LDL-c: Low density lipoprotein cholesterol; \\ MDL-LDL: Malondialdehyde-modified LDL; mLDL: Modified LDL; oxLDL-Ab: oxLDL \\ antibody; TG: Triglycerides
}

\section{Acknowledgments}

We thank profession Yu-tao Diao, the Institute of Basic Medicine of Shandong Academy of Medical Sciences for expert technical assistance with oxLDL-antibody test.

\section{Funding}

This study was funded by the fiftieth group of China Postdoctoral Science Foundation (No.2011M500747)

\section{Availability of data and materials}

Please contact author for data requests.

\section{Authors' contributions}

Members listed below made their substantial contributions to this manuscript. Hao Li and Da-qing Li design of this study, data analysis, and drafted the manuscript. Xiang-xin Li and Lu-qun Wang carried out the assay and the data and analysis, and checked the draft and performed the final approval of the version to be submitted. All authors read and approved the final manuscript.

\section{Competing interests}

The authors declare that they have no competing interests.

\section{Consent for publication}

All the co-authors and participants have given their consent for publication in Lipids in Health and Disease.

\section{Ethics approval and consent to participate}

The study design was ethically approved by the Medical Ethics Committee of Qilu Hospital of Shandong University approved the study protocol. All participants were provided written informed consent at the beginning of the study.

\section{Author details}

'Department of Hematology, Qilu Hospital, Shandong University, Jinan 250012, China. ${ }^{2}$ The Key Laboratory of Cardiovascular Remodelling and Function Research, Chinese Ministry of Education and Chinese Ministry of Health, Department of Cardiology, Qilu Hospital, Shandong University, 107\# Wenhuaxi Road, Jinan 250012, China.

Received: 3 August 2016 Accepted: 1 November 2016 Published online: 08 November 2016

\section{References}

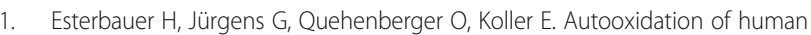
low density lipoprotein: loss of polyunsaturated fatty acids and vitamin $E$ and generation of aldehydes. J Lipid Res. 1987;28(5):495-509. PMID: 3598395.

2. Steinberg D, Parthasarathy S, Carew TE, Khoo JC, Witztum JL. Beyond cholesterol. Modifications of low-density lipoprotein that increase its atherogenicity. N Eng J Med. 1989;320(14):915-24. PMID:2648148.

3. Frostegård J, Huang YH, Rönnelid J, Schäfer-Elinder L. Platelet-activating factor and oxidized LDL induce immune activation by a common mechanism. Arterioscler Thromb Vasc Biol. 1997;17(5):963-8. PMID:9157962.
4. Tsimikas S, Brilakis ES, Lennon RJ, Miller ER, Witztum JL, McConnell JP, Kornman KS, Berger PB. Relationship of IgG and IgM autoantibodies to oxidized low density lipoprotein with coronary artery disease and cardiovascular events. J Lipid Res. 2007:48(2):425-33. PMID:17093289.

5. Ravandi A, Boekholdt SM, Mallat Z, Talmud PJ, Kastelein JJ, Wareham NJ, Miller ER, Benessiano J, Tedgui A, Witztum JL, et al. Relationship of lgG and IgM autoantibodies and immune complexes to oxidized LDL with markers of oxidation and inflammation and cardiovascular events: results from the EPIC-Norfolk Study. J Lipid Res. 2011;52(10):1829-36. PMID:21821825.

6. Hirsch HA, lliopoulos D, Joshi A, Zhang Y, Jaeger SA, Bulyk M, Tsichlis PN, Shirley Liu X, Struhl K. A transcriptional signature and common gene networks link cancer with lipid metabolism and diverse human diseases. Cancer Cell. 2010:17(14):348-61. PMID:20385360.

7. Chou MY, Fogelstrand L, Hartvigsen $K$, Hansen LF, Woelkers D, Shaw PX, Choi J, Perkmann T, Bäckhed F, Miller Yl, et al. Oxidation-specific epitopes are dominant targets of innate natural antibodies in mice and humans. J Clin Invest. 2009:119(5):1335-49. PMID:19363291.

8. Nguyen TG, McKelvey KJ, March LM, Hunter DJ, Xue M, Jackson CJ, Morris JM. Aberrant levels of natural IgM antibodies in osteoarthritis and rheumatoid arthritis patients in comparison to healthy controls. Immunol Lett. 2016; 170:27-36. PMID:26744098.

9. Qi X, De Stefano V, Su C, Bai M, Guo X, Fan D. Associations of antiphospholipid antibodies with splanchnic vein thrombosis: a systematic review with metaanalysis. Medicine (Baltimore). 2015;94(4):e496. PMID:25634200.

10. Hosseini H, Li Y, Kanellakis P, Tay C, Cao A, Tipping P, Bobik A, Toh BH, Kyaw T. Phosphatidylserine liposomes mimic apoptotic cells to attenuate atherosclerosis by expanding polyreactive lgM producing B1a lymphocytes. Cardiovasc Res. 2015;106(3):443-52. PMID:25681396.

11. Tsiantoulas D, Perkmann T, Afonyushkin T, Mangold A, Prohaska TA, PapacMilicevic N, Millischer V, Bartel C, Horkko S, Boulanger CM, et al. Circulating microparticles carry oxidation-specific epitopes and are recognized by natural IgM antibodies. J Lipid Res. 2015;56(2):440-8. PMID:25525116.

12. Moohebati M, Kabirirad V, Ghayour-Mobarhan M, Esmaily H, Tavallaie S, Akhavan Rezayat A, Pourghadamyari H, Sahebkar A. Investigation of serum oxidized lowdensity lipoprotein IgG levels in patients with angiographically defined coronary artery disease. Int J Vasc Med. 2014;2014:845960. PMID:24639897.

13. Zhang J, Wang $D$, He S. Roles of antibody against oxygenized low density lipoprotein in atherosclerosis: recent advances. Int J Clin Exp Med. 2015;8(8): 11922-9. PMID:26550105.

14. Brändlein S, Rauschert N, Rasche L, Dreykluft A, Hensel F, Conzelmann E, Müller-Hermelink HK, Vollmers HP. The human IgM antibody SAM-6 induces tumor-specific apoptosis with oxidized low-density lipoprotein. Mol Cancer Ther. 2007:6(1):326-33. PMID:17237291.

15. Diao Y, Li H, Li HQ, Zhou Y, Ma Q, Wang Y, Li D. Association of serum levels of lipid and its novel constituents with the different stages of esophageal carcinoma. Lipids Health Dis. 2009;8:48. PMID:19863824.

16. Allampallam K, Dutt D, Nair C, Shetty V, Mundle S, Lisak L, Andrews C, Ahmed $B$, Mazzone $L$, Zorat $F$, et al. The clinical and biologic significance of abnormal lipid profiles in patients with myelodysplastic syndromes. J Hematother Stem Cell Res. 2000;9(2):247-55. PMID:10813538.

17. Lanemo Myhrinder A, Hellqvist E, Sidorova E, Söderberg A, Baxendale H, Dahle C, Willander K, Tobin G, Bäckman E, Söderberg O, et al. A new perspective: molecular motifs on oxidized LDL, apoptotic cells, and bacteria are targets for chronic lymphocytic leukemia antibodies. Blood. 2008;111(7):3838-48. PMID: 18223168.

18. Li H, Diao YT, Li HQ, Ma Q, Cui J, Zhou YZ, Li D. The association between serum levels of oxLDL-lgG and oxLDL-lgM autoantibody with adult acute myeloblastic leukaemia. Lipids Health Dis. 2010;31:9-11 [PMID:20113525].

19. Aharon A, Levy Y, Bar-Dayan Y, Afek A, Zandman-Goddard G, Skurnik Y, Fabrrizzi F, Shoenfeld Y. Successful treatment of early secondary myelofibrosis in SLE with IVIG. Lupus. 1997;6(4):408-11. PMID:9175029.

20. Li H, Li XX, Ma Q, Cui J. The variability of oxLDL-induced cytotoxicity on different types of cell lines. Cell Biochem Biophys. 2013;67(2):635-44. PMID:23479334.

21. Fraley AE, Schwartz GG, Olsson AG, Kinlay S, Szarek M, Rifai N, Libby P, Ganz P. Witztum JL, Tsimikas S, et al. Relationship of oxidized phospholipids and biomarkers of oxidized low-density lipoprotein with cardiovascular risk factors, inflammatory biomarkers, and effect of statin therapy in patients with acute coronary syndromes: results from the MIRACL (Myocardial Ischemia Reduction with Aggressive Cholesterol Lowering) trial. J Am Coll Cardiol. 2009;53(23):2186-96. PMID:19497447. 
22. Dandapat A, Hu C, Sun L, Mehta JL. Small concentrations of ox-LDL induce capillary tube formation from endothelial cells via LOX-1-dependent redoxsensitive pathway. Arterioscler Thromb Vasc Biol. 2007;27(11):2435-42. PMID: 17717293.

23. Zabirnyk O, Liu W, Khalil S, Sharma A, Phang JM. Oxidized low-density lipoproteins upregulate proline oxidase to initiate ROS-dependent autophagy. Carcinogenesis. 2010;31(31):446-54. PMID:19942609.

24. Berliner JA, Navab M, Fogelman AM, Frank JS, Demer LL, Edwards PA, Watson AD, Lusis AJ. Atherosclerosis: basic mechanisms: oxidation, inflammation, and genetics. Circulation. 1995;91(9):2488-96. PMID:7729036.

25. Lopes-Virella MF, Virella G. Pathogenic role of modified LDL antibodies and immune complexes in atherosclerosis. J Atheroscler Thromb. 2013;20(10): 743-54 [PMID:23965492]

26. Amir S, Binder CJ. Experimental immunotherapeutic approaches for atherosclerosis. Clin Immunol. 2010;134(1):66-9. PMID:19666239.

27. Wolf D, Zirlik A, Ley K. Beyond vascular inflammation-recent advances in understanding atherosclerosis. Cell Mol Life Sci. 2015;72(20):3853-69. PMID: 26100516.

28. Virella G, Thorpe SR, Alderson NL, Derrick MB, Chassereau C, Rhett JM, Lopes-Virella MF. Definition of the immunogenic forms of modified human LDL recognized by human autoantibodies and by rabbit hyperimmune antibodies. J Lipid Res. 2004:45(10):1859-67. PMID:15258197.

29. Rossi GP, Cesari M, De Toni R, Zanchetta M, Maiolino G, Pedon L, Ganzaroli C, Maiolino P, Pessina AC. Antibodies to oxidized low-density lipoproteins and angiographically assessed coronary artery disease in white patients. Circulation. 2003;108(20):2467-72. PMID:14581399.

30. Tsimikas S, Bergmark C, Beyer RW, Patel R, Pattison J, Miller E, Juliano J, Witztum JL. Temporal increases in plasma markers of oxidized low-density lipoprotein strongly reflect the presence of acute coronary syndromes. J Am Coll Cardiol. 2003;41(3):360-70. PMID:12575961.

31. Nicolo D, Varadhachary AS, Monestier M. Atherosclerosis, antiphospholipid syndrome, and antiphospholipid antibodies. Front Biosci. 2007;12:2171-82. PMID:17127454.

32. Roselaar SE, Kakkanathu PX, Daugherty A. Lymphocyte populations in atherosclerotic lesions of apoE -/- and LDL receptor -/- mice: decreasing density with disease progression. Arterioscler Thromb Vasc Biol. 1996;16(8): 1013-8. PMID:8696940.

33. Pawlak K, Mysliwiec M, Pawlak D. Oxidized LDL to autoantibodies against oxLDL ratio - the new biomarker associated with carotid atherosclerosis and cardiovascular complications in dialyzed patients. Atherosclerosis. 2012; 224(1):252-7. PMID:22840691.

34. Masztalewicz M, Nowacki P, Kotlęga D, Bajer-Czajkowska A, Drechsler H. Anti-oxLDL antibodies are clinically insignificant for stroke patients. Neurol Res. 2014;36(1):86-91. PMID:24107551.

35. Khoo LH, Thiam CH, Soh SY, Angeli V. Splenic extrafollicular reactions and BM plasma cells sustain lgM response associated with hypercholesterolemia. Eur J Immunol. 2015;45(5):1300-12. PMID:25639537.

36. Charach G, Michowitz Y, Rogowski O, Charach L, Argov O, George J, Grosskopf I. Usefulness of antibodies to oxidized low-density lipoproteins as predictors of morbidity and prognosis in heart failure patients aged $\neq 65$ years. Am J Cardiol. 2015;116(9):1379-84. PMID:26372212.

37. Kumano-Kuramochi M, Fujimura T, Komba S, Maeda-Yamamoto M, Machida S. Screening, expression, and characterization of an anti-human oxidized low-density lipoprotein single-chain variable fragment. J Biosci Bioeng. 2016; 122:287-93.

\section{Submit your next manuscript to BioMed Central and we will help you at every step:}

- We accept pre-submission inquiries

- Our selector tool helps you to find the most relevant journal

- We provide round the clock customer support

- Convenient online submission

- Thorough peer review

- Inclusion in PubMed and all major indexing services

- Maximum visibility for your research

Submit your manuscript at www.biomedcentral.com/submit
C) Biomed Central 